\title{
CONSERVATION LAWS FOR SECOND-ORDER PARABOLIC PARTIAL DIFFERENTIAL EQUATIONS
}

\author{
B. VAN BRUNT ${ }^{1}$, D. PIDGEON ${ }^{1}$, M. VLIEG-HULSTMAN $^{1}$ and W. D. HALFORD ${ }^{1}$
}

(Received 2 January, 2002)

\begin{abstract}
Conservation laws for partial differential equations can be characterised by an operator, the characteristic and a condition involving the adjoint of the Fréchet derivatives of this operator and the operator defining the partial differential equation. This approach was developed by Anco and Bluman and we exploit it to derive conditions for second-order parabolic partial differential equations to admit conservation laws. We show that such partial differential equations admit conservation laws only if the time derivative appears in one of two ways. The adjoint condition, however, is a biconditional, and we use this to prove necessary and sufficient conditions for a certain class of partial differential equations to admit a conservation law.
\end{abstract}

\section{Introduction}

In this paper we study conservation laws for second-order parabolic partial differential equations (PDEs). In particular, given a PDE of the form

$$
u_{x x}-F\left(u, u_{x}, u_{t}\right)=0,
$$

we are interested in determining the class of functions $F$ that lead to conservation laws. Much of the research on conservation laws centres around applications of Noether's theorem, which requires the existence of a Lagrangian. Recently, however, results have been obtained for non-Lagrangian systems through a procedure developed by Anco and Bluman $[1,2]$. The advantage of this procedure is that, in the Lagrangian case, it bypasses the actual formulation of the Lagrangian, and more importantly, it is applicable to non-Lagrangian systems. The results in this section are based on the work of Anco and Bluman and Olver [4]. Although generalisations are available (see,

\footnotetext{
'Institute of Fundamental Sciences, Mathematics, Massey University, New Zealand; e-mail:

B.vanBrunt@massey.ac.nz,W.D.Halford@massey.ac.nz and M.Vlieg@massey.ac.nz.

(C) Australian Mathematical Society 2004, Serial-fee code 1446-1811/04
} 
for example, Anco and Bluman) we limit our discussion to scalar functions of two independent variables and scalar PDEs. In this section we introduce some basic results and notation. The account is intended to be primarily descriptive, and the notation follows that used by Olver.

Let $f: \Omega \rightarrow \mathbb{R}$ be a function of two independent variables $x$ and $t$ defined in some region $\Omega \subseteq \mathbb{R}^{2}$, and let $u^{(k)}$ denote the set of $k^{\text {th }}$-order derivatives of $u$. Thus $u^{(1)}=$ $\left\{u_{x}, u_{t}\right\}, u^{(2)}=\left\{u_{x x}, u_{x t}, u_{t}\right\}$, et cetera. We use the notation $\left(x, t, u, u^{(1)}, \ldots, u^{(n)}\right)$ to denote the vector consisting of $x, t, u$ and all the derivatives of $u$ up to and including the $n^{\text {th }}$-order derivatives. We shall assume here that $u$ is in the function space $C^{q}(\Omega)$, where $q$ is some integer sufficiently large so that any partial derivatives required exist and are continuous.

Recall that the $n^{\text {th }}$ prolongation of a function $u$ is defined as

$$
p r^{(n)} u=\left\{u, u^{(1)}, \ldots, u^{(n)}\right\}
$$

for example,

$$
p r^{(2)} u=\left\{u, u^{(1)}, u^{(2)}\right\}=\left\{u, u_{x}, u_{t}, u_{x x}, u_{x t}, u_{t t}\right\}
$$

Let $U \subseteq C^{q}(\Omega)$. The $n$-jet space associated with $M=\Omega \times U$ will be denoted by $M^{(n)}$. This space has elements of the form $\left(x, t, u, u^{(1)}, \ldots, u^{(n)}\right)$, that is, $(x, t) \times p r^{(n)} u$.

Let $H: U \rightarrow C^{0}(\Omega)$ be an $n^{\text {th }}$-order differential operator. The operator $H$ may also be regarded as a function defined on $M^{(n)}$, and we thus use the notation $H[u]=H\left(x, t, u, u^{(1)}, \ldots, u^{(n)}\right)$ where convenient. We shall assume that $H$ is smooth with respect to the variables in $M^{(n)}$. In this section, we are concerned with conservation laws associated with the PDE

$$
H[u]=0 .
$$

Recall that a conservation law for (1.2) is a relation of the form

$$
\nabla \cdot \mathbf{P}[u]=D_{x} P_{1}[u]+D_{t} P_{2}[u]=0,
$$

which is satisfied for any solution $u$ to (1.2). Here $\mathbf{P}=\left(P_{1}, P_{2}\right)$ represents the conserved flux and density respectively, and the $P_{k}: U \rightarrow C^{1}(\Omega)$ are generally either partial differential operators or integro-differential operators. In (1.3), $D_{x}$ and $D_{t}$ denote the total derivative operators with respect to $x$ and $t$ respectively.

If (1.3) is a conservation law for (1.2), then it can be shown $([4$, p. 266]) that there exists an operator $Q$ such that

$$
\nabla \cdot \mathbf{P}[u]=Q[u] H[u] .
$$

The operator $Q$ is called the characteristic of the conservation law. The characteristic is said to be trivial if $Q[u]=0$ for all solutions to (1.2); otherwise it is called non-trivial. 
Conservation laws are usually determined via Noether's theorem. In order to apply this result the PDE (1.2) needs to have a Lagrangian formulation and to be reformulated as a variational problem involving some functional $K: U \rightarrow \mathbb{R}$ of the form

$$
K[u]=\int_{\Omega} L[u] d x d t .
$$

The operator $L$ is a partial differential operator called the Lagrangian. Noether's theorem requires the construction of a Lagrangian $L$ such that any solution to (1.2) corresponds to a stationary value for the functional $K$. A necessary condition for a function $u \in U$ to yield a stationary value for $K$ is satisfaction of the Euler-Lagrange equation

$$
E(L[u])=0,
$$

where $E$ is the Euler operator defined by

$$
E=\sum_{J}(-D)_{,} \frac{\partial}{\partial u_{J}} .
$$

Here $J$ is a multi-index that takes arguments such as $0, x, t, x x, x t, t t, x x x$ et cetera, with $u_{0}=u$, and for $J=k_{1} k_{2} \cdots k_{m}\left(k_{j}=x\right.$ or $\left.t\right)$,

$$
D_{J}=D_{k_{1}} D_{k_{2}} \cdots D_{k_{m}}
$$

and

$$
(-D)_{J}=(-1)^{m} D_{k_{1}} D_{k_{2}} \cdots D_{k_{m}} .
$$

The partial derivatives in the definition of $E$ are with respect to the jet space variables. For example, if $L[u]=1+u u_{x}^{2}+u_{t}^{2}+u_{x t} u_{t}$, then

$$
\begin{aligned}
E(L[u]) & =\sum_{J}(-D)_{j} \frac{\partial}{\partial u_{J}} L[u] \\
& =\frac{\partial L}{\partial u}-D_{x} \frac{\partial L}{\partial u_{x}}-D_{t} \frac{\partial L}{\partial u_{t}}+D_{x} D_{t} \frac{\partial L}{\partial u_{x t}} \\
& =u_{x}^{2}-2 u_{x}^{2}-2 u u_{x x} .
\end{aligned}
$$

Noether's theorem requires the PDE $H[u]=0$ to be identified as the Euler-Lagrange equation for some variational problem.

Noether's theorem connects conservation laws with variational symmetries. These symmetries are defined as a connected group of transformations with infinitesimal generators

$$
X=\xi_{1}(x, t, u) \frac{\partial}{\partial x}+\xi_{2}(x, t, u) \frac{\partial}{\partial t}+\phi(x, t, u) \frac{\partial}{\partial u},
$$


such that the prolongation operator $X^{p r(n)}$, defined by

$$
X^{p r(n)}=\xi_{1}(x, t, u) \frac{\partial}{\partial x}+\xi_{2}(x, t, u) \frac{\partial}{\partial t}+\sum_{J}\left(D_{J} \phi(x, t, u)\right) \frac{\partial}{\partial u_{J}}
$$

where $|J| \leq n$, satisfies the equation

$$
X^{p r(n)}(L[u])+L[u] \nabla \cdot \xi=0
$$

for all $\left(x, t, u, u^{(1)}, \ldots, u^{(n)}\right) \in M^{(n)}$. Here $\xi=\left(\xi_{1}(x, t, u), \xi_{2}(x, t, u)\right)$. Now if $Q$ is a characteristic of a variational symmetry, that is, $X_{Q}=Q[u] \partial / \partial u$, where $Q[u]=\phi-\xi_{1} u_{x}-\xi_{2} u_{t}$, then Noether's theorem indicates that $Q$ is also a characteristic of a conservation law. In this manner the determination of conservation laws is reduced to the determination of variational symmetries. Olver [4] and Bluman and Kumei [3] discuss Noether's theorem in more depth. The point is that in this formulation one must first derive a Lagrangian in order to determine the symmetries, and this can prove computationally awkward. Note that, in general, an operator $H$ need not have a Lagrangian so that Noether's theorem is applicable to only a special class of operators.

The method developed by Anco and Bluman relies on two results, the proofs of which can be found in Olver. The first result concerns the rôle of null-Lagrangians in the theory. An operator $L$ defined on the space $M^{n}$ is a null-Lagrangian if $E(L[u])=0$ for all $u \in U$. The following theorem indicates that the kernel of the Euler operator is the space of total divergences.

THEOREM 1.1. An operator $L$ is a null-Lagrangian if and only if there is an operator $\mathbf{P}=\left(P_{1}, P_{2}\right)$ such that $L[u]=\nabla \cdot \mathbf{P}[u]$, for all $u \in U$.

Suppose now that $\nabla \cdot \mathbf{P}[u]=0$ is a conservation law for (1.2). Then there is a $Q$ such that $Q[u] H[u]=\nabla \cdot \mathbf{P}[u]$ and since $E(L[u])=0$ for all $u \in U$ we have that

$$
\begin{aligned}
E(Q[u] H[u]) & =\sum_{J}(-D)_{J} \frac{\partial}{\partial u_{J}}(Q[u] H[u]) \\
& =\sum_{J}(-D)_{J}\left(\frac{\partial H}{\partial u_{J}} Q\right)+\sum_{J}(-D)_{J}\left(\frac{\partial Q}{\partial u_{J}} H\right)=0 .
\end{aligned}
$$

The last calculation brings to the fore operators corresponding to Fréchet derivative adjoints. Briefly, the Fréchet derivative $D_{H}$ of an operator $H$ acting on some operator $Q$ is given by

$$
D_{H}(Q)=\sum_{J}\left(\frac{\partial H}{\partial u_{J}}\right) D_{J} Q
$$


and the adjoint of $D_{H}$, denoted by $D_{H}^{\#}$, is given by

$$
D_{H}^{\#}(Q)=\sum_{J}(-D)_{J}\left(\frac{\partial H}{\partial u_{J}} Q\right)
$$

For example, if $H[u]=H\left(u, u_{x}, u_{t}, u_{x t}\right)$, then

and

$$
D_{H}(Q)=\frac{\partial H}{\partial u} Q+\frac{\partial H}{\partial u_{x}} D_{x} Q+\frac{\partial H}{\partial u_{t}} D_{t} Q+\frac{\partial H}{\partial u_{x t}} D_{x} D_{t} Q
$$

$$
D_{H}^{\#}(Q)=\frac{\partial H}{\partial u} Q-D_{x}\left(\frac{\partial H}{\partial u_{x}} Q\right)-D_{t}\left(\frac{\partial H}{\partial u_{t}} Q\right)+D_{x} D_{t}\left(\frac{\partial H}{\partial u_{x t}} Q\right) .
$$

In terms of the adjoints of the Fréchet derivatives $D_{H}$ and $D_{Q}$, the condition

$$
E(Q[u] H[u])=0
$$

is equivalent to

$$
D_{H}^{\#}(Q)+D_{Q}^{\#}(H)=0 .
$$

Now, it is well-known that if $H[u]$ corresponds to some Euler-Lagrange equation then the Fréchet derivative $D_{H}$ is self-adjoint, that is, $D_{H}=D_{H}^{\#}$, and for this case we recover the familiar equation

$$
D_{H}(Q)+D_{Q}^{\sharp}(H)=0
$$

which is a necessary and sufficient condition for $Q$ to be the characteristic of a variational symmetry (see, for example, [4, p. 333]). The equation governing a conservation law, however, is (1.7) (not (1.8)) and it is certainly possible that this equation can be satisfied regardless of whether a Lagrangian formulation is available. The next result $([4$, p. 333$])$ shows that $(1.7)$ characterises conservation laws.

THEOREM 1.2. An operator $Q$ is a characteristic of a conservation law for the PDE $H[u]=0$ if and only if (1.7) is satisfied for all $u \in U$.

Armed with Theorem 1.2, it is possible to generate conservation laws without appealing to Noether's theorem directly. Suppose for example $Q[u] H[u]$ represents a conservation law for (1.2). Then for any solution $u$ to (1.2) we have that $D_{Q}^{\#}(H[u])=0$ and consequently the operator $Q$ must satisfy the condition

$$
D_{H}^{\#}(Q[u])=0
$$

for any solution $u$ to (1.2). Note that if $D_{H}$ is self-adjoint, then (1.9) reduces to the condition that $Q$ is either a point or generalised symmetry of (1.2), though not 
necessarily a variational symmetry. If $D_{H}$ is not self-adjoint we can still use (1.9) to determine the possible operators leading to characteristics of a conservation law. As with the self-adjoint case, there is no guarantee that a solution to (1.9) gives a characteristic to a conservation law because (1.7) must then be satisfied for all $u \in U$ and not just for solutions to $H[u]=0$.

Once a characteristic for a conservation law has been identified, there exist formulæ for extracting the corresponding conservation law. A general formula based on homotopy invariance is given in Anco and Bluman [1,2]. In practice, this formula can prove formidable to implement, but it is often possible to deduce the conservation law by more elementary means (for example, integration by parts).

Theorem 1.2 is particularly interesting because it is a biconditional. Suppose that $H[u]=0$ has a conservation law $\nabla \cdot \mathbf{P}[u]=0$. Then there is some characteristic $Q$ such that $\nabla \cdot \mathbf{P}[u]=Q[u] H[u]$. Now the operators $Q$ and $H$ must satisfy (1.7) for all $u \in U$ by Theorem 1.2. The existence of a conservation law thus places some restrictions directly on the operator $H$. In the next section we use this observation to deduce the forms a second-order parabolic PDE must take in order to have a conservation law of prescribed order.

\section{Parabolic equations}

Consider a parabolic PDE of the form (1.1). We assume that $F$ is a function twice continuously differentiable with respect to the indicated arguments and that

$$
F_{u_{i}} \neq 0,
$$

so that (1.1) does not reduce to an ordinary differential equation.

Suppose that there exists a conservation law for the PDE and let $Q$ be a corresponding characteristic. At this stage we must place some restrictions on the highest order derivatives appearing in the characteristic. This restriction limits the highest order derivatives appearing in the equation $\nabla \cdot \mathbf{P}[u]=0$. Suppose for definiteness that we limit our investigation to conservation laws with smooth first-order characteristics. This means that the operator $Q$ may be regarded as a smooth function of $x, t, u, u_{x}$ and $u_{t}$ and we thus write $Q[u]=Q\left(x, t, u, u_{x}, u_{t}\right)$. We will assume that $Q$ is a non-trivial characteristic.

The Fréchet derivative of $H$ is

$$
D_{H}(Q)=D_{x}^{2} Q-F_{u_{x}} D_{x} Q-F_{u_{1}} D_{t} Q-F_{u} Q,
$$

so that the associated adjoint operator is

$$
D_{H}^{*}(Q)=D_{x}^{2} Q+D_{x}\left(F_{u_{x}} Q\right)+D_{t}\left(F_{u_{t}} Q\right)-F_{u} Q .
$$


Note that in general $D_{H}^{*} \neq D_{H}$, so that (1.1) is not, in general, an Euler-Lagrange equation. Similarly,

$$
D_{Q}^{\#}(H)=-D_{x}\left(Q_{u_{x}} H\right)-D_{l}\left(Q_{u_{t}} H\right)+Q_{u} H .
$$

The operators $Q$ and $H$ must satisfy (1.7), and substituting the above operators into this equation gives (after a tedious calculation) the relation

$$
W_{1} u_{x x}+W_{2} u_{x t}+W_{3} u_{x t}^{2}+W_{4} u_{t t}+W_{5}=0,
$$

which must be satisfied for all $u \in U$. Here

$$
\begin{aligned}
& W_{1}=Q_{x u_{x}}+u_{x} Q_{u u_{x}}-Q_{t u_{t}}-u_{t} Q_{u u_{t}}+2 Q_{u}+(F Q)_{u_{s} u_{x}}, \\
& W_{2}=Q_{x u_{t}}+u_{x} Q_{u u_{t}}+(F Q)_{u_{x} u_{t}}, \\
& W_{3}=Q_{u_{t} u_{t}} \\
& W_{4}=(F Q)_{u_{t} u_{t}}
\end{aligned}
$$

and

$$
\begin{aligned}
W_{s}= & u_{x}^{2} Q_{u u}+u_{x}\left(2 Q_{x u}+(F Q)_{u u_{x}}\right)+u_{t}(F Q)_{u u_{t}}+Q_{x x} \\
& +(F Q)_{x u_{x}}+(F Q)_{t u_{t}}-(F Q)_{u} .
\end{aligned}
$$

Since (2.4) must be satisfied for all $u \in U$ we may regard $x, t, u$ and the derivatives of $u$ as jet-space variables. Now, the only term in (2.4) containing $u_{x x}$ is $W_{1} u_{x x}$ and hence we have that $W_{1}=0$. Similar arguments indicate that $W_{k}=0$ for $k=2,3,4$ and these equations imply $W_{5}=0$. These five equations place restrictions on $Q$ and $F$. Theorem 1.2 implies that satisfaction of these equations is a necessary and sufficient condition for $F$ to have a conservation law with characteristic $Q$.

The equation $W_{3}=0$ implies that $Q$ must be of the form

$$
Q=A u_{\imath}+B,
$$

where $A$ and $B$ are functions of $x, t, u$ and $u_{x}$. The equations $W_{3}=0$ and $W_{4}=0$ imply

$$
\begin{aligned}
(F Q)_{u_{t} u_{t}} & =F_{u_{t} u_{t}} Q+2 F_{u_{t}} Q_{u_{t}}+F Q_{u_{t} u_{t}} \\
& =F_{u_{t} u_{t}} Q+2 F_{u_{t}} Q_{u_{t}},
\end{aligned}
$$

and (2.1) and (2.5) imply that this condition is equivalent to

$$
\frac{F_{u_{1} u_{t}}}{F_{u_{t}}}=-\frac{2 A}{A u_{t}+B} \text {. }
$$


There are two cases to consider. These cases correspond to whether or not $Q$ depends on $u_{t}$.

Suppose that $A \neq 0$. Since $F$ does not depend explicitly on the variables $x$ and $t$, the quotient $F_{u_{t}} / F_{u_{u} u_{t}}$ does not depend explicitly on these variables; hence the quotient $B / A$ depends only on $u$ and $u_{x}$. Let $C=B / A$. Equation (2.6) implies that $F$ must be of the form

$$
F=\frac{K}{u_{t}+C}+E
$$

where $K$ and $E$ are functions of only $u$ and $u_{x}$.

Suppose that $A=0$. Then

$$
Q=B\left(x, t, u, u_{x}\right),
$$

and (2.6) implies that $F_{u_{t} u_{t}}=0$ so that $F$ is of the form

$$
F=R u_{t}+S,
$$

where $R$ and $S$ are functions of only $u$ and $u_{x}$.

It is of interest to note that if the PDE (1.1) has a conservation law then the derivative $u_{\text {t }}$ can appear in the equation in only two different ways. More formally, we have the following result.

THEOREM 2.1. If the PDE (1.1) has a conservation law with a non-trivial smooth first-order characteristic $Q$ then either $F$ is of the form (2.7), in which case $Q$ is of the form (2.5); or $F$ is of the form (2.9), in which case $Q$ is of the form (2.8).

The above theorem provides a necessary but not sufficient condition for a secondorder parabolic PDE to admit a conservation law. If $F$ is of one of the forms specified in the theorem, there is no guarantee that the corresponding PDE has a conservation law since $F$ and $Q$ must still satisfy the remaining equations

$$
\begin{aligned}
W_{1}= & Q_{x u_{x}}+u_{x} Q_{u u_{t}}-Q_{t u_{t}}-u_{t} Q_{u u_{t}}+2 Q_{u}+(F Q)_{u_{x} u_{x}}=0, \\
W_{2}= & Q_{x u_{t}}+u_{x} Q_{u u_{t}}+(F Q)_{u_{s} u_{t}}=0, \\
W_{5}= & u_{x}^{2} Q_{u u}+u_{x}\left(2 Q_{x u}+(F Q)_{u u_{t}}\right) \\
& +u_{t}(F Q)_{u u_{t}}+Q_{x x}+(F Q)_{x u_{x}}+(F Q)_{t u_{t}}-(F Q)_{u}=0 .
\end{aligned}
$$

The above equations place restrictions on functions such as $A$ and $B$ that arise from the integrations to get the forms for $Q$ and $F$. If the functions introduced through these integrations satisfy these equations then Theorem 2.1 shows that the corresponding PDE has a conservation law. Equations (2.10)-(2.12), however, provide less tractable 
information about the form of $Q$ and $F$. In fact, the characterisation problem is underdetermined with regard to the dependence of $F$ on $u$ and $u_{x}$. There is considerable freedom in this system. We illustrate this by a simple example.

Suppose that $F$ is of the form (2.7). Suppose that we specify the characteristic as

$$
Q=u_{t}+C
$$

where $C$ is a given function and that we also specify that $E=0$. We will show that even after these functions are prescribed there exists a $K$ such that the PDE

$$
H[u]=u_{x x}-\frac{K}{u_{t}+C}=0
$$

has a conservation law. Now,

$$
\begin{aligned}
Q[u] H[u] & =u_{x x} u_{t}+C\left(u, u_{x}\right) u_{x x}-K\left(u, u_{x}\right) \\
& =D_{x}\left(u_{x} u_{t}\right)-D_{t}\left(u_{x}^{2} / 2\right)+C\left(u, u_{x}\right) u_{x x}-K\left(u, u_{x}\right) .
\end{aligned}
$$

The above expression can certainly be written in a divergence form if $K$ is chosen so that $C u_{x x}-K$ is a perfect differential. Let $M_{u_{x}}=C$. We need only to find a function $K$ so that for $M_{u}=-K / u_{x}$ the compatibility condition

$$
\frac{\partial C}{\partial u}=-\frac{\partial}{\partial u_{x}}\left(\frac{K}{u_{x}}\right)
$$

is satisfied. Evidently we may choose

$$
K\left(u, u_{x}\right)=-u_{x} \int \frac{\partial C}{\partial u} d u_{x}
$$

For example, if $C=u u_{x}+u^{2}$, then we can choose

$$
K=-u_{x} \int\left(u_{x}+2 u\right) d u_{x}=-\frac{u_{x}^{3}}{2}-2 u u_{x}^{2} .
$$

Then $M=u u_{x}^{2} / 2+u^{2} u_{x}$ and the conservation law is

$$
Q H=\nabla\left(u_{x} u_{t}+M, u_{x}^{2} / 2\right)=0 .
$$

\section{A characterisation of parabolic equations}

The results of Section 2 can be used to determine certain classes of PDEs that admit conservation laws. Here, we focus on PDEs of the form

$$
H[u]=u_{x x}-a(u) u_{1}-b(u) u_{x}-c(u)=0,
$$


where $a, b$ and $c$ are smooth functions of $u$, but it is clear that the method can be applied to more general cases. The problem is to find necessary and sufficient conditions on the functions $a, b$ and $c$ so that the PDE (3.1) has a non-trivial conservation law. We retain assumption (2.1) so that in this analysis

$$
a(u) \neq 0,
$$

and we restrict our attention to smooth first-order characteristics.

It is clear that any PDE of the form (3.1) satisfies the conditions of Theorem 2.1 since it is of the form (2.9), where $R=a(u)$ and $S=b(u) u_{x}+c(u)$. The PDE (3.1) has a conservation law with a first-order characteristic if and only if the characteristic is of the form (2.8), that is, independent of $u_{t}$, and the functions $a, b$ and $c$ are such that $(2.10)-(2.12)$ are satisfied.

Since $Q_{u_{t}}=0,(2.11)$ implies $(F Q)_{u_{x} u_{t}}=\left(F_{u_{t}} Q\right)_{u_{x}}=a(u) Q_{u_{x}}=0$, and (3.2) thus implies that $Q_{u_{x}}=0$, that is, $Q=Q(x, t, u)$. Equation (2.10) implies $Q_{u}=0$, and hence $Q=Q(x, t)$. Equation (2.12) implies that

$$
Q_{x x}+a(u) Q_{t}+b(u) Q_{x}-c^{\prime}(u) Q=0 .
$$

We thus have the following result.

THEOREM 3.1. A PDE of the form (3.1) has a conservation with a non-trivial smooth first order characteristic if and only if $Q=Q(x, t)$ and the functions $Q, a, b$ and $c$ are such that (3.3) is satisfied for all $x, t$ and $u$.

We now study the conditions that (3.3) places on $Q, a, b$ and $c$ in detail. Equation (3.3) must be satisfied for all $x, t$ and $u$, and differentiating this expression with respect to $u$ and dividing by $Q$ gives

$$
a^{\prime}(u)(\ln Q)_{t}+b^{\prime}(u)(\ln Q)_{x}-c^{\prime \prime}(u)=0 .
$$

Differentiating (3.4) with respect to $x$ gives

$$
a^{\prime}(u)(\ln Q)_{x t}+b^{\prime}(u)(\ln Q)_{x x}=0,
$$

and differentiating (3.4) with respect to $t$ gives

$$
a^{\prime}(u)(\ln Q)_{t r}+b^{\prime}(u)(\ln Q)_{x t}=0 .
$$

Equations (3.5) and (3.6) highlight four cases characterised by whether $a^{\prime}(u)$ and/or $b^{\prime}(u)$ vanish.

Case 1: $a^{\prime}(u) \neq 0, b^{\prime}(u) \neq 0$. Suppose that $a^{\prime}(u) \neq 0$ and $b^{\prime}(u) \neq 0$. Then (3.5) and (3.6) imply that either

$$
(\ln Q)_{x t}=(\ln Q)_{x x}=(\ln Q)_{t t}=0
$$


or

$$
\frac{a^{\prime}(u)}{b^{\prime}(u)}=-\frac{(\ln Q)_{x x}}{(\ln Q)_{x t}}=-\frac{(\ln Q)_{x t}}{(\ln Q)_{t t}}
$$

Subcase 1a. Suppose that (3.7) is satisfied. Then $\ln Q$ must be of the form $\ln Q=\alpha t+\beta x+\gamma$, where $\alpha, \beta$ and $\gamma$ are constants; hence

$$
Q=\Upsilon e^{\alpha \tau+\beta x},
$$

where $\Upsilon$ is a nonzero constant.

Equation (3.4) implies

$$
\alpha a^{\prime}(u)+\beta b^{\prime}(u)-c^{\prime \prime}(u)=0,
$$

that is,

$$
\alpha a(u)+\beta b(u)=c^{\prime}(u)+\delta,
$$

where $\delta$ is a constant of integration. Equation (3.4) shows that $\delta=0$ and therefore

$$
\alpha a(u)+\beta b(u)=c^{\prime}(u) .
$$

Subcase 1b. Suppose that (3.8) is satisfied. Since $a$ and $b$ depend only on $u$ and $Q$ depends only on $x$ and $t$ there is a constant $\Lambda_{1}$ such that

$$
\frac{a^{\prime}(u)}{b^{\prime}(u)}=\Lambda_{1} \text {. }
$$

Equations (3.5) and (3.11) imply

$$
\frac{c^{\prime \prime}}{b^{\prime}}=\Lambda_{1}(\ln Q)_{t}+(\ln Q)_{x},
$$

and consequently there is a constant $\Lambda_{2}$ such that

$$
\frac{c^{\prime \prime}(u)}{b^{\prime}(u)}=\Lambda_{2} \text {. }
$$

Hence for this case the functions $a, b$ and $c$ satisfy the relations

$$
\begin{aligned}
a(u) & =\Lambda_{1} b(u)+k_{1}, \\
c^{\prime}(u) & =\Lambda_{2} b(u)+k_{2} .
\end{aligned}
$$

We can obtain the characteristic by noting first that (3.12) and (3.13) imply

$$
\Lambda_{1}(\ln Q)_{t}+(\ln Q)_{x}=\Lambda_{2} .
$$


Equations (3.3), (3.14) and (3.15) imply

$$
Q_{x x}+k_{1} Q_{t}+k_{2} Q_{x}+\frac{b(u)}{Q}\left(\Lambda_{1}(\ln Q)_{t}+(\ln Q)_{x}-\Lambda_{2}\right)=0
$$

hence, by (3.16),

$$
Q_{x x}+k_{1} Q_{t}+k_{2} Q_{x}=0
$$

The $Q_{t}$ term in the above PDE can be eliminated using (3.16) and thus

$$
Q_{x x}+\lambda_{1} Q_{x}+\lambda_{2} Q_{x}=0
$$

where $\lambda_{1}=-k_{1} / \Lambda_{1}, \lambda_{2}=k_{2}-\Lambda_{2} / \Lambda_{1}$. Hence, assuming that $\lambda_{1}^{2}-4 \lambda_{2} \neq 0$,

$$
Q(x, t)=A(t) e^{\mu_{1} x}+B(t) e^{\mu_{2} x},
$$

where the constants $\mu_{k}$ are the (possibly complex) roots of $\mu^{2}+\lambda_{1} \mu+\lambda_{2}=0$. Substituting expression (3.19) into (3.17) gives

$$
e^{\mu_{1} x}\left(k_{1} A^{\prime}(t)+\left(\mu_{1}^{2}+k_{2}\right) A(t)\right)+e^{\mu_{2} x}\left(k_{1} B^{\prime}(t)+\left(\mu_{2}^{2}+k_{2}\right) B(t)\right)=0,
$$

which must be true for all $x$ and $t$. Since $\mu_{1} \neq \mu_{2}$, we have

$$
A(t)=\beta_{1} e^{-\left(\mu_{1}^{2}+k_{2}\right) t / k_{1}} \quad \text { and } \quad B(t)=\beta_{2} e^{-\left(\mu_{2}^{2}+k_{2}\right) t / k_{1}},
$$

where $\beta_{j}$ are constants. Note that if $\lambda_{1}^{2}-4 \lambda_{2}=0$, so that $\mu_{1}=\mu_{2}$, we could still determine $Q$ using the basis functions $e^{\mu_{1} x}$ and $x e^{\mu_{1} x}$.

Case 2: $\boldsymbol{a}^{\prime}(u) \neq 0, \boldsymbol{b}^{\prime}(\boldsymbol{u})=\mathbf{0}$. Suppose that $a^{\prime}(u) \neq 0$ and $b(u)=b=$ constant. Equation (3.4) implies that there is a constant $\Lambda$ such that

$$
c^{\prime \prime} / a^{\prime}=\Lambda_{1}(\ln Q)_{t}=\Lambda
$$

hence

$$
c^{\prime}(u)=\Lambda a(u)+k,
$$

where $k$ is a constant. Equation (3.5) indicates that $\ln Q$ is of the form

$$
\ln Q=h_{1}(t)+h_{2}(x),
$$

and (3.20) shows that $h_{1}(t)=\Lambda t+\eta$, where $\eta$ is a constant. The characteristic is thus of the form

$$
Q=\Upsilon e^{\Lambda t+h_{2}(x)},
$$


where $\Upsilon$ is a non-zero constant. Substituting this expression into (3.3) and using relation (3.21) gives the Riccati equation

$$
h_{2}^{\prime \prime}(x)+\left(h_{2}^{\prime}\right)^{2}+b h_{2}^{\prime}-k=0,
$$

which, assuming $b^{2}+4 k \neq 0$, has the general non-trivial solution

$$
h_{2}^{\prime}(x)=\frac{\alpha_{1} \beta_{1} e^{\beta_{1} x}+\alpha_{2} \beta_{2} e^{\beta_{2} x}}{\alpha_{1} e^{\beta_{1} x}+\alpha_{2} e^{\beta_{2} x}} .
$$

Here $\alpha_{j}$ are arbitrary constants (not both zero) and $\beta_{j}$ are the (possibly complex) solutions to the equation $\beta^{2}+b \beta-k=0$. (If $b^{2}+4 k=0$, then $\beta_{1}=\beta_{2}$ and we can replace the function $e^{\beta_{2} x}$ with $x e^{\beta_{1} x}$ and its derivative to get a general solution. If $k=0$ then the trivial solution $h^{\prime}=0$ is a possibility, but even this solution leads to a non-trivial characteristic.) In any event, the characteristic is determined up to integration constants by (3.22) and (3.23).

Case 3: $a^{\prime}(u)=0, b^{\prime}(u) \neq 0$. Suppose that $a(u)$ is a non-zero constant $a$ and that $b^{\prime}(u) \neq 0$. The calculations for this case are similar to those for Case 2 . For this case we have

$$
c^{\prime}(u)=\Lambda b(u)+k,
$$

where $\Lambda$ and $k$ are constants, and it can be shown that the characteristic must be of the form

$$
Q=\Upsilon e^{\left(k-\Lambda^{2}\right) t / a+\Lambda x},
$$

where $\Upsilon$ is a non-zero constant.

Case 4: $\boldsymbol{a}^{\prime}(\boldsymbol{u})=\mathbf{0}, \boldsymbol{b}^{\prime}(\boldsymbol{u})=\mathbf{0}$. Suppose that $a(u)=a$ and $b(u)=b$ where $a$ and $b$ are constants with $a \neq 0$. In this case (3.4)-(3.6) are satisfied and place no restrictions on $Q$. Equation (3.3), however, shows that $c^{\prime \prime}(u)=0$ so that $c$ must be of the form $c(u)=k_{1} u+k_{2}$, where $k_{1}$ and $k_{2}$ are constants. Equation (3.3) also implies that any non-trivial solution $Q$ to the linear PDE

$$
Q_{x x}+a Q_{1}+b Q_{x}-k_{1} Q=0
$$

corresponds to the characteristic of a conservation law.

The analysis of the above cases indicates that in all but Case 4, the characteristics are determined up to integration constants and that they can be determined explicitly. If we distil the conditions on the functions $a, b$ and $c$ from the above calculations we see that there are in fact only two possibilities. These conditions are summarised in the following theorem. 
THEOREM 3.2. The PDE (3.1) has a conservation law with a non-trivial first order characteristic if and only if there exist constants $\alpha, \beta, \gamma$ and $\delta$ such that either the equation

$$
\alpha a(u)+\beta b(u)=c^{\prime}(u)
$$

or the system

$$
\begin{aligned}
\alpha a(u) & =b(u)+\gamma \\
c^{\prime}(u) & =\beta b(u)+\delta,
\end{aligned}
$$

is satisfied for all $u \in U$.

We finish this section with some examples that recover well-known results and illustrate the above analysis.

EXAMPLE 1. Any equation of the form $u_{x x}-a(u) u_{t}-b(u) u_{x}-c=0$, where $c$ is a constant, has a conservation law. We can choose $\alpha=\beta=0$ so that (3.27) is satisfied. Indeed, we can construct the conservation law directly without appealing to the analysis, namely, $\nabla \cdot\left(u_{x}-N(u)-c x,-M(u)\right)=0$, where $N^{\prime}(u)=a(u)$ and $M^{\prime}(u)=b(u)$.

EXAMPLE 2 (Fisher's Equation). Fisher's equation is $u_{x x}-\kappa\left(u_{t}-u+u^{2}\right)=0$, where $\kappa$ is a non-zero constant. Here $a(u)=\kappa, b(u)=0$ and $c(u)=\left(-u+u^{2}\right) \kappa$. Since $c^{\prime}(u)=(2 u-1) \kappa$ there is no choice of constants $\alpha$ and $\beta$ such that (3.27) is satisfied for all $u$. Since $b(u)=0$ and $c^{\prime}$ is not a constant, there are no constants to satisfy (3.29). Hence Fisher's equation cannot have a conservation law with a first order characteristic.

EXAMPLE 3 (Burgers' Equation). Burgers' equation is $u_{x x}-\kappa\left(u_{t}+u u_{x}\right)=0$, where $\kappa$ is a non-zero constant. Here $a(u)=\kappa, b(u)=\kappa u$ and $c(u)=0$. We know from Example 1 that certainly one conservation law is the equation in divergence form, that is, $\nabla \cdot\left(u_{x}-\kappa u^{2} / 2,-\kappa u\right)=0$. Burgers' equation corresponds to Case 3 , and we can glean more information from the characteristic. Specifically, since $c^{\prime}=0$ we know from (3.24) that $\Lambda=k=0$. This means that the characteristic $Q$ must be constant ((3.25)). The relationship (1.4) thus implies that the only conservation law for Burgers' equation with a first-order characteristic is the equation in divergence form.

EXAMPLE 4 (The Heat Equation). Consider the heat equation $u_{x x}-u_{t}=0$. Clearly this equation has the conservation law

$$
\nabla \cdot\left(u_{x},-u\right)=0
$$


but, in contrast with Burgers' equation, there are many other possibilities. The heat equation is included in case 4 and any solution to the PDE

$$
Q_{x x}+Q_{t}=0
$$

will produce a characteristic for a conservation law. The special choice $Q=$ constant gives the conservation law (3.30). For the general case, if $Q$ is a solution to the PDE (3.31) and $u$ is a solution to the heat equation then

$$
Q H=\nabla \cdot\left(Q u_{x}-Q_{x} u,-Q u\right)=0,
$$

so that we can readily derive the conservation law given the solution $Q$.

Steinberg and Wolf [5] studied conservation laws for the heat equation and derived the PDE (3.31) as a condition for the functional $J[u]=\int_{-\infty}^{\infty} Q(x, t) u(x, t) d x$ to be constant in time under the boundary conditions

$$
\left.\left(Q u_{x}-Q_{x} u\right)\right|_{-\infty} ^{\infty}=0
$$

Given this restriction on the solutions $u$ and $Q$ the invariance of $J$ with respect to time follows immediately from integration with respect to $x$ of the conservation law (3.32), assuming that the order of differentiation and integration can be interchanged. For instance, the solution $Q=x$ produces the first moment $J[u]=\int_{-\infty}^{\infty} x u(x, t) d x$, which is constant in time for all solutions to the heat equation satisfying the boundary conditions $\left.\left(x u_{x}-u\right)\right|_{-\infty} ^{\infty}=0$. A more general class of solutions to the PDE (3.31) is given by the "heat polynomials"

$$
Q(x, t)=v_{n}(x,-t)=t^{n / 2} H_{n}\left(\frac{x}{2 \sqrt{t}}\right)
$$

where $H_{n}$ denotes a Hermite polynomial. Each of these solutions produces a distinct conservation law, and linear combinations of these solutions produce yet more. Steinberg and Wolf [5] arrive at the relationship between conserved densities and the heat polynomials by exploiting symmetry operators of the heat equation. They use the resulting conservation laws to characterise the time dependence of all the moments of solutions to the heat equation.

\section{References}

[1] S. C. Anco and G. W. Bluman, "Derivation of conservation laws from nonlocal symmetries of differential equations”, J. Math. Phys. 37 (1996) 2361-2375. 
[2] S. C. Anco and G. W. Bluman, "Direct construction of conservation laws from field equations", Phys. Rev. Lett. 78 (1997) 2869-2873.

[3] G. W. Bluman and S. Kumei, Symmetries and differential equations (Springer, New York, 1989).

[4] P. J. Olver, Applications of Lie groups to differential equations (Springer, New York, 1986).

[5] S. Steinberg and K. B. Wolf, "Symmetry, conserved quantities and moments in diffusive equations", J. Math. Anal. Appl. 80 (1981) 36-45. 